\title{
Optimizing Motheye Nanostructures and Achieving 100\% Transmission
}

\author{
Behnam Kheyraddini Mousavi', Morteza Rezaei Talarposhti² \\ ${ }^{1}$ Department of Electromechanical Engineering, Northern New Mexico College, Espanola, USA \\ ${ }^{2}$ Department of Chemical Engineering, University of California Irvine, Irvine, USA \\ Email: behnam.mousavi@nnmc.edu,mrezaeit@uci.edu
}

How to cite this paper: Mousavi, B.K. and Talarposhti, M.R. (2020) Optimizing Motheye Nanostructures and Achieving 100\% Transmission. Journal of Applied Mathematics and Physics, 8, 229-239. https://doi.org/10.4236/jamp.2020.82018

Received: January 3, 2020

Accepted: January 19, 2020

Published: January 22, 2020

Copyright (c) 2020 by author(s) and Scientific Research Publishing Inc. This work is licensed under the Creative Commons Attribution International License (CC BY 4.0).

http://creativecommons.org/licenses/by/4.0/ (c) (i) Open Access

\begin{abstract}
Nanostructured Antireflections Layers (NALs) are a promising substitute for conventional antireflection layers. In the nature, such structures have long existed on insects' eyes. Here, we have derived a general form of mathematical relation that generates a profile close to that of surface of a Moth's eye. By properly designing NALs over dielectric, light has been efficiently transmitted through our high index nanostructures with minimal reflection. The parameters determining the transmission efficiency are the pitch and the profile of the periodic nanostructures. By optimizing the profile, we have designed NALs with transmission reaching $100 \%$. Our periodic structures have minimal dependence on the pitch. This makes it possible to fabricate NALs with the very broadband transmission, without inducing diffraction to lower wavelengths of the transmitted light. Also, our periodic NALs make it possible to transmit laser lights without scattering.
\end{abstract}

\section{Keywords}

Maximal Transmission, Broadband, Reflection, Laser, Nano-Structure

\section{Introduction}

Conventional antireflections consist of one or more layers over each other laying over a substrate. These layers work based on destructive interference of light [1] [2] [3]. While one layer can only be used for one specific wavelength or angle of illumination, multi-layer structures partially solve this limitation [1] [3] [4]. However, still, layers have rather low bandwidth and angular tolerance. A major problem with this technology arises from the residual stress between these layers or layers and substrate [4]. This lowers the lifetime of layered antireflection coatings when they're used in environments with fluctuating temperatures. Also, 
when a high-power beam of light passes through them, these layers peel off the substrate [5] due to the difference in the expansion coefficient of different materials. Recently, nanotechnology and nanostructures [6] [7] [8] [9] have solved many problems in technology introducing materials [10] that exhibit special characteristics electrically, mechanically [11]-[15] and when they're under illuminations of light [6] [7] [16] [17]. Fabrication method of these nanomaterials [6] [18] [19] usually depends on the type of the materials and the physical characteristics they're designed for.

NALs are the new generation of anti-reflection coatings designed to deal with previous problems [6]. These nanostructured materials are designed based on a fill factor concept [1]. This means that the effective refractive index depends on the volume occupied by the nanostructure [17]. Such coatings are widely used for increasing light transmission to solar cells [20] [21], and light extraction from LED's [22] [23] and lasers [24] [25]. Sub-wavelength NAL structures are usually carved out of substrate for smoother transition of refractive index. NALs are used for different applications. For example, these structures can be used for increasing transmission in infrared detectors [26] or transmission of laser through different optical elements like lenses or polarizers [6] [17]. For solar cells and LEDs, the goal is to enhance scattering by eliminating total internal reflection or enhancing near-surface reflection by scattering light off normal [20] [21] [27].

Nanostructuring the surfaces of dielectric materials is often used to improve transmission at the silicon/air interface in IR applications. As previously mentioned, one of the main applications of NALs is enhancing transmission of light through optical elements used in high-power-laser applications [25] [28] [29] [30]. In such applications, it is also crucial to avoid scattering. Therefore, the randomly distributed NALs are replaced by orderly ones [25] [30] [31]. Although periodic structures don't impose scattering to the transmitted light, usually such structure's max efficiency happens at a certain pitch for every specific wavelength [17]. To the best of our knowledge, the researchers who have designed these nanostrucutres, first calculated refractive index of different $2 \mathrm{D}$ antireflection films laying over each other for maximal transmission. Afterwards, they related the calculated refractive indexes to the amount filled by a $3 \mathrm{D}$ dielectric disk using fill factor or Maxwell-Garnett approximation [17]. Although the 2D layers in some cases result in $100 \%$ transmission, due to the approximation, the 3D geometries associated with these 2D structures do not yield the same max transmission [17]. One way to deal with this problem is directly designing the NALs and evaluating the transmission of light through the 3D design using numerical methods.

In nature already nanostructures exist that maximize light transmission into the eyes of some insects. A series of mathematical equations are fitted to a motheye structure. We've seen that for high index materials like silicon, the transmission through ordinary Motheye nanostructures falls below 100\%. Using Rigorous Coupled Wave Analysis (RCWA) we have evaluated the profiles that fit 
the moth's eye. Then, we have further optimized these nanostrucutres for enhancing light transmission through high index materials like silicon using more complex mathematical expressions for the profile. In this paper, using RCWA we have explored Motheye like optimized nanostructures of Silicon, for reaching $100 \%$ transmission (silicon is transparent in IR). This is a big achievement for high power laser applications used in industry where even a tenth of a percent back reflection is considerable [17] [25] [30] [31] and can be destructive. In applications such as transparency glasses, even a percent of glare can have a drastic effect on reduction of quality of image [32]. Our structures have eliminated even the tiniest reflection making them very suitable for a wide range of applications. In our previous work [6], we observed that logarithmically designed NALs efficiency, highly depended on pitch. Therefore, for gaining high transmission for higher wavelengths we had to increase the pitch. However, our current Motheye nanostructures are relatively insensitive to the pitch. Therefore keeping the pitch constant (submicron), we have evaluated the nanostructures transmissions. This is another plus for the periodic nanostructures efficiency which is usually dependant on the pitch [17]. By designing nanostructures with sub-micron pitch, we can design very broadband NALs which transmit both high and low wavelengths, without imposing diffraction to lower wavelengths.

\section{Maximizing Transmission}

In this section, we have investigated multiple profiles for periodic nanostructures to reach $100 \%$ transmission. In our previous work, we investigated profiles which generated over up to $99.1 \%$ for a height equal to $\pi$ phase shift [6]. Here, we find the profiles for maximal transmission of light, even superior to the previous profiles [6]. The heights of NALs will generate the same phase shift in the transmitted electromagnetic waves for a fair comparison. We try different mathematical equations for the profile of NALs and evaluate each profile using RCWA. The equations we use have a general form of $f=1-\left(\frac{x}{p}\right)^{n_{1}}$, where $f$ represents the fill factor and $\frac{x}{p}$ represents side length of cross section at each elevation normalized to pitch. We have chosen this mathematical equation to its resemblance to motheye. By swiping $n_{1}$, we have gained series of mathematical relations resulting in $100 \%$ transmission. As the first step, we assume that $n_{2}=1$. We find the suitable pitch for maximizing transmission at roughly $12 \mu \mathrm{m}$ numerically ( Pitch $=0.64 \mu \mathrm{m}$ ) using Bisection method [33]. At this pitch, if we assume $n_{1}=1$, the transmission will be maximum around $12 \mu \mathrm{m}$ wavelength. Then we keep the pitch constant and swipe the wavelength from $0.95 \mu \mathrm{m}$ to 25 $\mu \mathrm{m}$. The transmission is shown in Figure 1. The max transmission of $100 \%$ happens at $n_{1}=0.7$. However, the transmission drops rapidly for longer wavelengths compared to cases with higher $n_{1}$ values. Note that by changing $n_{1}$ the wavelength that maximum transmission happens deviates slightly. 


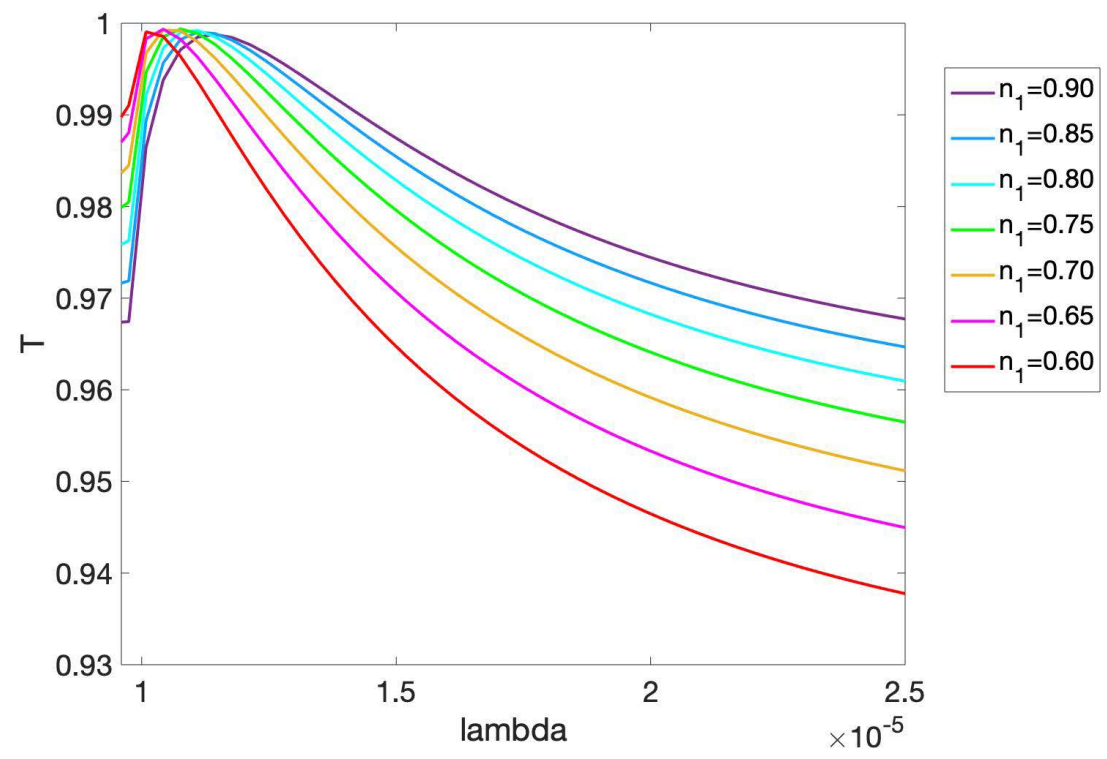

Figure 1. " $n_{1}$ ” changes from 0.6 to 0.9 . The pitch is kept constant at $0.64 \mu \mathrm{m}$. It's clear that the max point deviates from $\lambda=4 \mu \mathrm{m}$ by changing $n_{1}$. The max transmission is for $n_{1}=0.7$ where the peak transmission is $99.94 \%$. The max bandwidth belongs to $n_{1}=0.9$.

For further improving the profile, we generalize the mathematical relation to $\left(f=\left(1-(x / m)^{n_{1}}\right)^{n_{2}}\right)$. It is seen that if $n_{2} \neq 1$, the max point doesn't happen at 0.7 anymore. In Figure 2(a), the maximum 100\% happens for $n_{2}=0.9$ (in part (a) $\left.n_{1}=0.6\right)$. For Figure 2(b), the $\max 99.99 \%$ happens for $n_{2}=0.85$ and $n_{2}=0.90$ (in part (b) $n_{1}=0.7$ ). In Figure 2(c), $\max 100 \%$ happens for $n_{2}=0.7, n_{2}=0.75, n_{2}=0.80, n_{2}=0.85$ (in part (c) $n_{1}=0.8$ ). The bandwidths in Figure 2 are shown in Table 1. Further investigation has shown that the max transmission bandwidth happens for $n_{1}=0.75$. For $n_{1}=0.75$, we have swiped $n_{2}$ between 0.55 and 0.75 .

In Figure 3, the transmission is shown for a wavelength span from $9.5 \mu \mathrm{m}$ to $25 \mu \mathrm{m}$. We further reduce $n_{2}$ and increase the wavelength span to find how much the performance drops at wavelengths even longer than $25 \mu \mathrm{m}$. Keep in mind that in designing the NALs the height of the pillar is set to a $\pi$ phase shift.

In Figure 4, $\left(n_{1}=0.75\right)$, the max transmission is $100 \%$ which happens for $n_{2}=0.55$ and $n_{2}=0.50$. However, at longer wavelengths (above $50 \mu \mathrm{m}$ ), the highest transmission is $99.82 \%$ for $n_{2}=0.50$ and $n_{2}=0.45$ (Figure $4(\mathrm{a})$ ). Our simulations show that for $n_{1}=0.65$, although one would not get a $100 \%$ transmission, the spectrum of transmission is better than the previous case. Figure 4 (b) shows that when $n_{2}=0.45$, the transmission reduces to $99.96 \%$ for high wavelengths (above $50 \mu \mathrm{m}$ ).

The importance of the profiles introduced is that their max transmission is not dependant on pitch. This is a big advantage compared to our previous work [6], where the transmission peak for each wavelength happened at a specific 


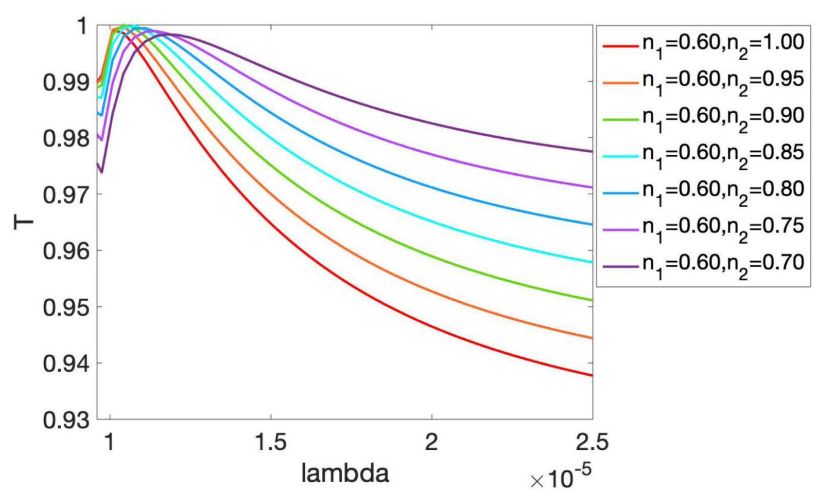

(a)

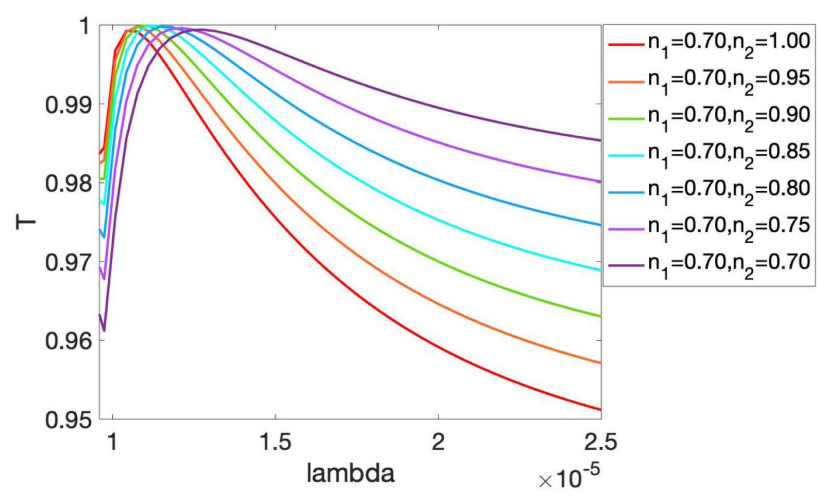

(b)

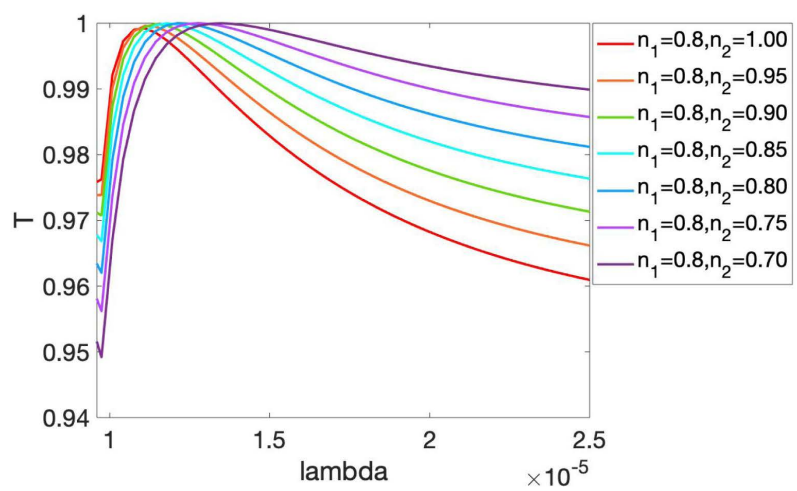

(c)

Figure 2. In all the three figures, $n_{2}$ is changing from 0.7 to 1 . (a) $n_{1}=0.6$ (b) $n_{1}=0.7$ (c) $n_{1}=0.8$.

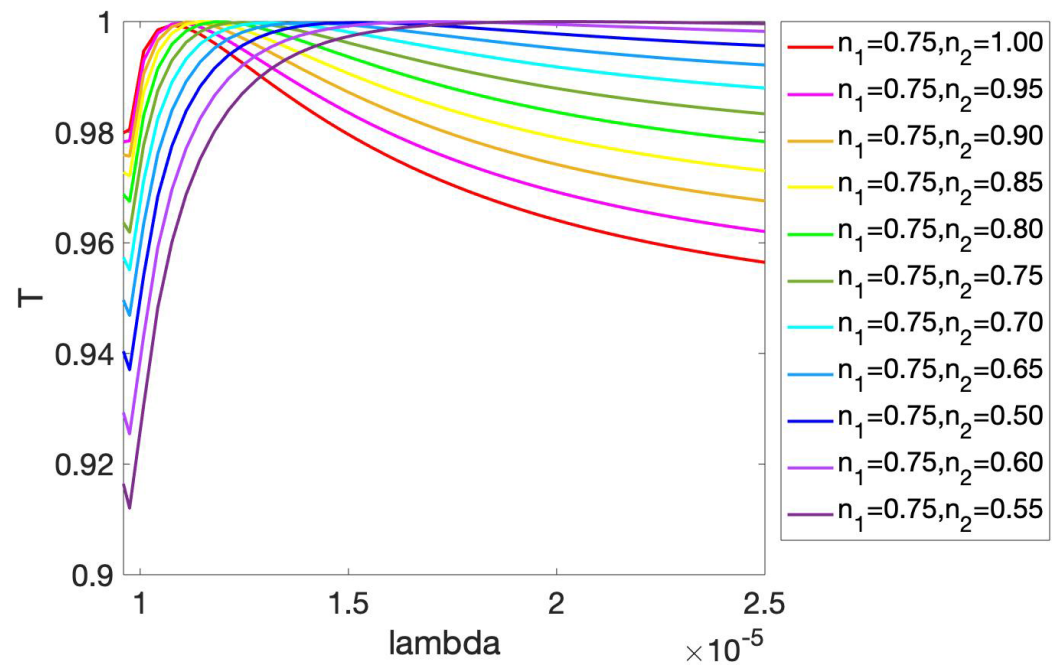

Figure 3. NALs designed based on $n_{1}=0.75$ show the max bandwidth specially at lower powers of $n_{2}$.

pitch, linearly related to the wavelength. In that case, the max transmission for each wavelength happened at a specific pitch, therefore one would need to change the pitch of NALs for maximizing different wavelengths. This is not desired since for gaining high transmission at longer wavelengths, one would need 


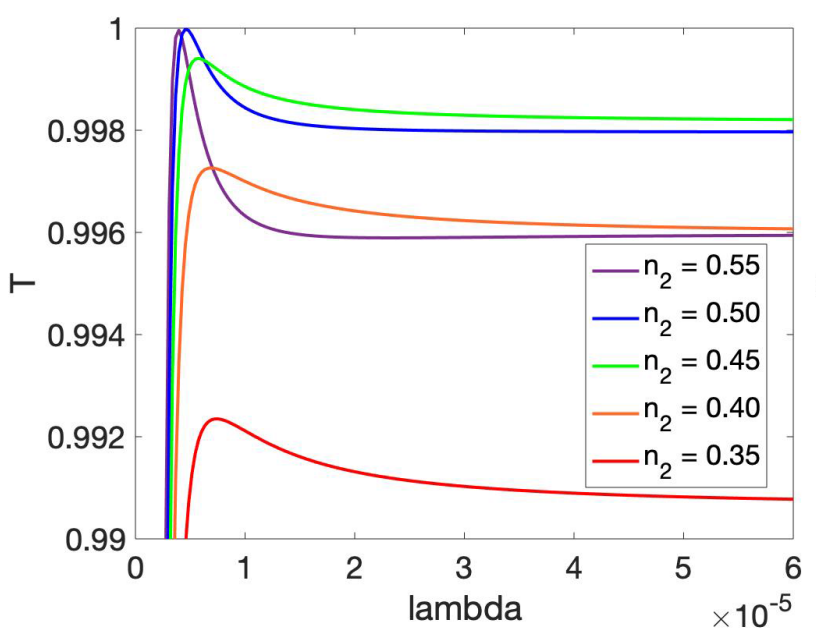

(a)

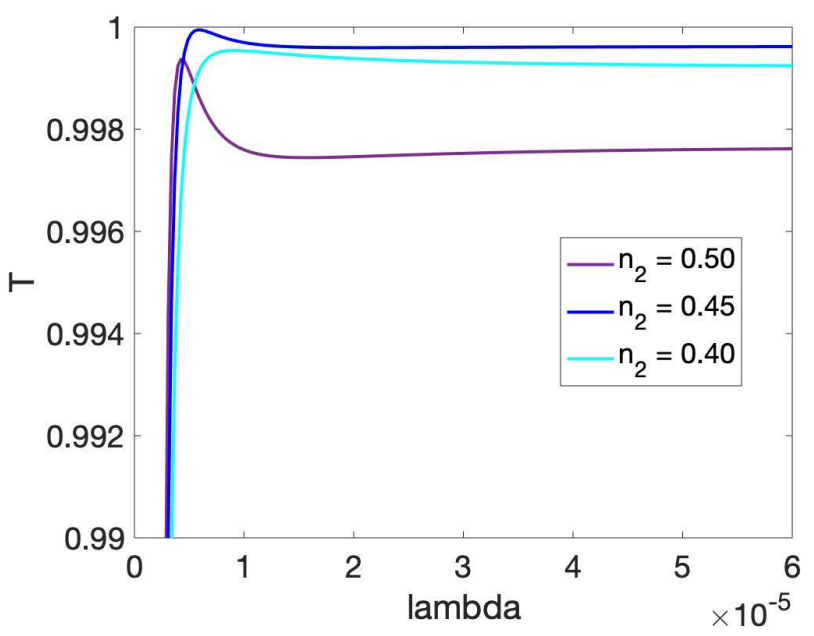

(b)

Figure 4. Better spectral transmission (a) The spectrum related to " $n_{1}=0.75$ ” for $n_{2}$ between 0.35 to 0.55 . (b) The spectrum related to " $n_{1}=0.65$ ” for $n_{2}$ between 0.4 to 0.50 .

Table 1. Transmission Band-width for three cases of Figure 2 based on $99 \%$ transmission cut-off.

\begin{tabular}{cccc}
\hline Index of refraction & $n_{1}=0.60$ & $n_{1}=0.70$ & $n_{1}=0.80$ \\
\hline$n_{2}=0.70$ & $5 \mu \mathrm{m}$ & $8.5 \mu \mathrm{m}$ & $13.6 \mu \mathrm{m}$ \\
$n_{2}=0.75$ & $4.2 \mu \mathrm{m}$ & $6.6 \mu \mathrm{m}$ & $9.3 \mu \mathrm{m}$ \\
$n_{2}=0.80$ & $3.5 \mu \mathrm{m}$ & $5 \mu \mathrm{m}$ & $6.8 \mu \mathrm{m}$ \\
$n_{2}=0.85$ & $2.8 \mu \mathrm{m}$ & $4.2 \mu \mathrm{m}$ & $5.5 \mu \mathrm{m}$ \\
$n_{2}=0.90$ & $2.4 \mu \mathrm{m}$ & $3.5 \mu \mathrm{m}$ & $4.5 \mu \mathrm{m}$ \\
$n_{2}=0.95$ & $2.0 \mu \mathrm{m}$ & $2.9 \mu \mathrm{m}$ & $3.8 \mu \mathrm{m}$ \\
$n_{2}=1.00$ & $1.7 \mu \mathrm{m}$ & $2.4 \mu \mathrm{m}$ & $3.4 \mu \mathrm{m}$ \\
\hline
\end{tabular}

to linearly increase the pitch of the nanostructure. Then, if we want to use the nanostructure for both longer and shorter wavelengths, diffraction will be inevitable for shorter wavelengths due to higher pitch. The limit of pitch for complete elimination of diffraction is shown in Equation (1) $\left(\lambda_{i n c}\right.$ is the wavelength of incidence).

$$
\text { Pitch } \leq \frac{\lambda_{\text {inc }}}{2 n_{\text {sub }}}
$$

The RCWA approach shows that our new profile is not pitch dependant to a very good extend. This is to our belief related to the way it distributes the E-field in space above the nanostructure. The spacial distribution of wave inside and out the NALs is subject of a subsequent publication which we are currently working on using finite element method. The refractive index of the nanostructures is shown in Figure 5. The refractive index is related to the profile at each elevation using fill-factor concept. The mathematical relation relating the two is shown in Equation (2) and Equation (3). 


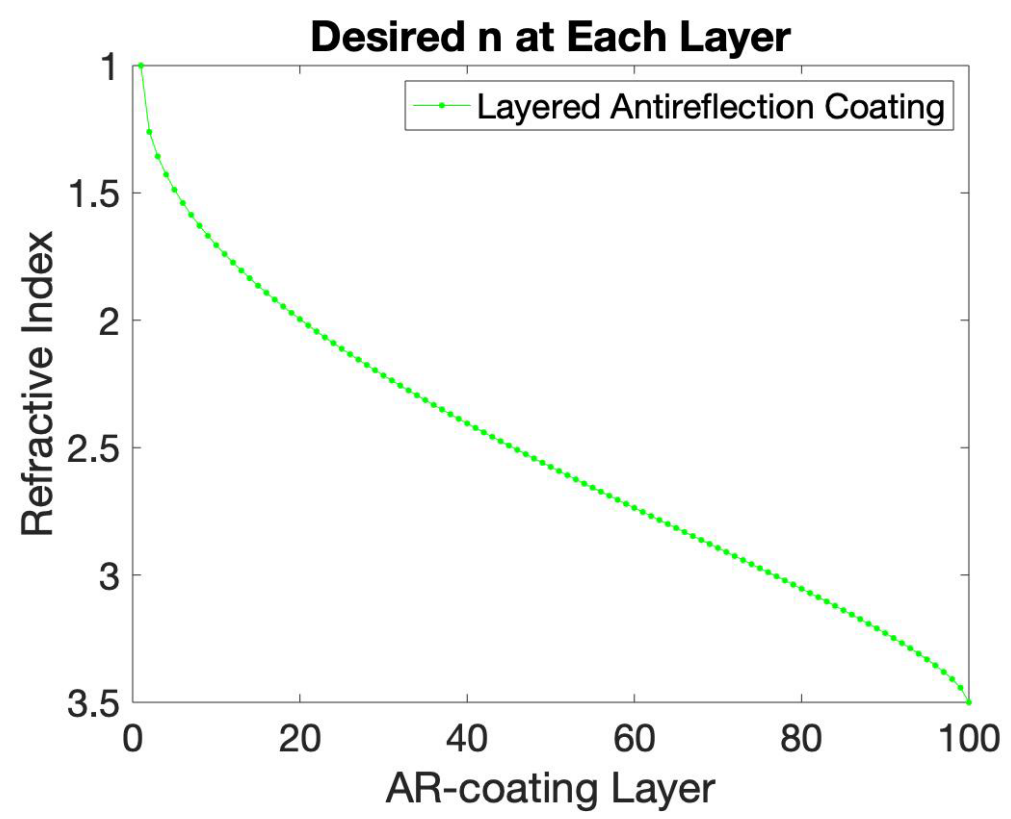

Figure 5. The refractive index related to the Motheye nanostructures at each elevation for $n_{1}=0.6$ and $n_{2}=0.45$.

$$
\begin{aligned}
\text { FillFactor } & =A \times n_{S i}+(1-A) \times n_{A i r} \\
x & =\sqrt{\text { Fill-Factor }}
\end{aligned}
$$

In the equation, we have assumed that the volume is occupied by $S i$, but in general it can be any material that is transparent at the illumination wavelength. Previously, we had found the proper refractive index at each elevation from substrate and assigned a geometric profile to the calculated index. Here, we first find a proper profile by mimicking nature and then related to a refractive index at each elevation.

In Equation (3), $x$ is length or width of the cross-section of NAL. We have not yet been able to fabricate a 3-D NAL with square cross-section from tip to base. Therefore, we have fitted a geometry with circular cross-section, to the square cross-section. The side-view and 3D profile of the periodic structures are shown normalized to the pitch in Figure 6. The resulting structures resemble that of motheye surface and can be considered "Enhanced Motheye-NanoStructures".

\section{Conclusion}

NALs are a promising option for enhancing transmission of light from air into high index materials. By engineering the profile of the NALs, one can reach very high transmissions. These structures offer zero residual stress with the substrate when exposed to high power laser beams and are of significant importance for transmitting light energy into high-index substrates. Our nanostructures yield a $100 \%$ transmission. This high transmission at different wavelengths has negligible dependence on the pitch of the nanostructure. This is another achievement compared to our previous design. Previously for each wavelength, we needed to 


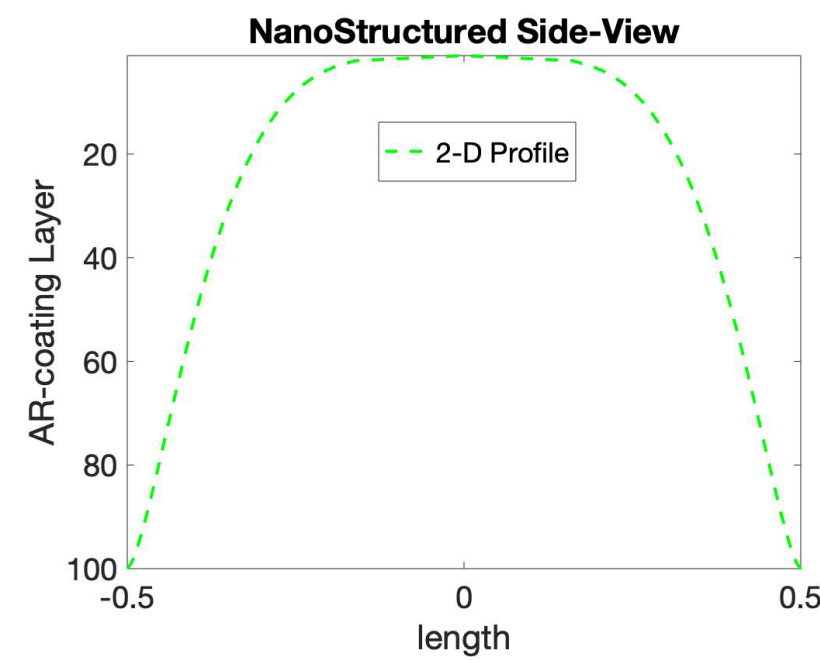

(a)

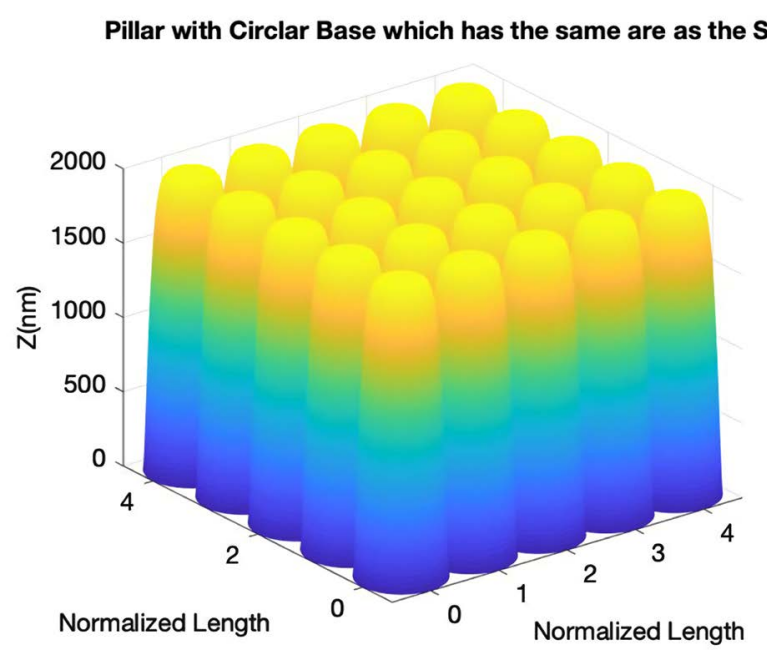

(b)

Figure 6. Motheye like profiles with very high performance are shown. (a) 2-D cross-section consisted of 100 layers (b) 3D profile of the periodic motheye structures normalized to the pitch with circular cross-section.

change the pitch of the nanostructures to maintain a high transmission. Therefore, NALs designed for longer wavelengths can have small pitches (even below $1 \mu \mathrm{m}$ ). This is important if one wants the nanostructures to function at lower wavelengths alongside the longer wavelengths. Our broadband nanostructures do not impose diffraction to the shorter wavelengths (due to very small pitch), while they highly enhance transmission for above $50 \mu \mathrm{m}$ wavelengths. This makes our NALs highly desirable for transmitting laser lights at a broad span of wavelengths. The profiles of these nanostructures resemble that of a motheye's surface, and considering the capabilities and designing approach, they can be considered enhanced motheye nanostructures.

\section{Acknowledgements}

This work is done with the funding of Center for High Technology Materials at University of New Mexico.

\section{Conflicts of Interest}

The authors declare no conflicts of interest regarding the publication of this paper.

\section{References}

[1] Raut, H.K., Ganesh, V.A., Nair, A.S. and Ramakrishna, S. (2011) Anti-Reflective Coatings: A Critical, in-Depth Review. Energy \& Environmental Science, 4, 3779-3804. https://doi.org/10.1039/clee01297e

[2] Bouhafs, D., Moussi, A., Chikouche, A. and Ruiz, J. (1998) Design and Simulation of Antireflection Coating Systems for Optoelectronic Devices: Application to Silicon Solar Cells. Solar Energy Materials and Solar Cells, 52, 79-93. https://doi.org/10.1016/S0927-0248(97)00273-0

[3] Zhao, J., Wang, A., Altermatt, P. and Green, M. (1995) Twenty-Four Percent Effi- 
cient Silicon Solar Cells with Double Layer Antireflection Coatings and Reduced Resistance Loss. Applied Physics Letters, 66, 3636-3638. https://doi.org/10.1063/1.114124

[4] Askar, K., Phillips, B.M., Fang, Y., Choi, B., Gozubenli, N., Jiang, P. and Jiang, B. (2013) Self-Assembled Self-Cleaning Broadband Anti-Reflection Coatings. Colloids and Surfaces A: Physicochemical and Engineering Aspects, 439, 84-100. https://doi.org/10.1016/j.colsurfa.2013.03.004

[5] Xu, Y., Zhang, B., Fan, W.H., Wu, D. and Sun, Y.H. (2003) Sol-Gel Broadband Anti-Reflective Single-Layer Silica Films with High Laser Damage Threshold. Thin Solid Films, 440, 180-183. https://doi.org/10.1016/S0040-6090(03)00828-9

[6] Mousavi, B.K., Mousavu, A.K., Busani, T., Zadeh, M.H. and Brueck, S. (2019) Nanostructured Anti-Reflection Coatings for Enhancing Transmission of Light. Journal of Applied Mathematics and Physics, 7, 3083-3100.

https://doi.org/10.4236/jamp.2019.712217

[7] Kheyraddini-Mousavi, A., Leseman, Z. and Kheyraddini-Mousavi, B. (2019) High Speed Michelson Interferometer Microscope. US Patent No. 10,436,570.

[8] Liu, J., Talarposhti, M., Asset, T., Sabarirajan, D.C., Parkinson, D.Y., Atanassov, P. and Zenyuk, I.V. (2019) Understanding the Role of Interfaces for Water Management in PGM-Free Electrodes in Polymer Electrolyte Fuel Cells. ACS Applied Energy Materials, 2, 3542-3553. https://doi.org/10.1021/acsaem.9b00292

[9] Roy, A., Talarposhti, M.R., Normile, S.J., Zenyuk, I.V., De Andrade, V., Artyushkova, K., Serov, A. and Atanassov, P. (2018) Nickel-Copper Supported on a Carbon Black Hydrogen Oxidation Catalyst Integrated into an Anion-Exchange Membrane Fuel Cell. Sustainable Energy \& Fuels, 2, 2268-2275. https://doi.org/10.1039/C8SE00261D

[10] Karbassian, F., Mousavi, B.K., Rajabali, S., Talei, R., Mohajerzadeh, S. and Asl-Soleimani, E. (2014) Formation of Luminescent Silicon Nanowires and Porous Silicon by Metal-Assisted Electroless Etching. Journal of Electronic Materials, 43, 1271-1279. https://doi.org/10.1007/s11664-014-3051-3

[11] Mousavi, A.K., Alaie, S. and Leseman, Z.C. (2016) Basic MEMS Actuators. In: Encyclopedia of Nanotechnology, Springer, Amsterdam, 1-16. https://doi.org/10.1007/978-94-007-6178-0_292-2

[12] Mousavi, A.K. and Leseman, Z.C. (2012) Basic MEMS Actuators. In: Encyclopedia of Nanotechnology, Springer, Amsterdam, 173-185.

[13] Mousavi, A., Kashamolla, M. and Leseman, Z. (2013) Improved Model for the Adhesion of $\mu$ Cantilevers: Theory and Experiments. Journal of Micromechanics and Microengineering, 23, Article ID: 115011. https://doi.org/10.1088/0960-1317/23/11/115011

[14] Parizi, K.B., Peyvast, N., Mousavi, B.K., Mohajerzadeh, S. and Fathipour, M. (2010) Schottky Barrier Nano-MOSFET with an Asymmetrically Oxidized Source/Drain Structure. Solid-State Electronics, 54, 48-51.

https://doi.org/10.1016/j.sse.2009.09.010

[15] Mousavi, A.K., Atwater, M.A., Mousavi, B.K., Jalalpour, M., Taha, M.R. and Leseman, Z.C. (2014) Mechanical and Electrical Characterization of Entangled Networks of Carbon Nanofibers. Materials, 7, 4845-4853. https://doi.org/10.3390/ma7064845

[16] Mousavi, B.K., Mousavi, A.K., Hieber, T.J., Chen, J. and Leseman, Z.C. (2019) Mode II Adhesion Energy Analysis of Stiction-Failed Poly-Si Cantilevers Using a MEMS Load Cell. Journal of Micromechanics and Microengineering, 29, Article ID: 075013. 
https://doi.org/10.1088/1361-6439/ab173e

[17] Mousavi, B.K. (2018) Selected Applications of Silicon Nanopillar Arrays. Ph.D. Thesis, the University of New Mexico, Albuquerque, NM.

[18] Mousavi, A.K., Abbas, K., Elahi, M.M.M., Lima, E., Moya, S., Butner, J.D., Pinon, D., Benga, A., Mousavi, B.K. and Leseman, Z.C. (2014) Pulsed Vacuum and Etching Systems: Theoretical Design Considerations for a Pulsed Vacuum System and Its Application to XeF 2 Etching of Si. Vacuum, 109, 216-222. https://doi.org/10.1016/j.vacuum.2014.07.028

[19] Kheyraddini Mousavi, B., Behzadirad, M., Silani, Y., Karbasian, F., Kheyraddini Mousavi, A. and Mohajerzadeh, S. (2019) Metal-Assisted Chemical Etching of Silicon and Achieving Pore Sizes as Small as $30 \mathrm{~nm}$ by Altering Gold Thickness. Journal of Vacuum Science \& Technology A: Vacuum, Surfaces, and Films, 37, Article ID: 061402. https://doi.org/10.1116/1.5112776

[20] Kumar, A., Chaliyawala, H., Siddhanta, S. and Barshilia, H.C. (2016) Broadband Quasi-Omnidirectional Subwavelength Nanoporous Antireflecting Surfaces on Glass Substrate for Solar Energy Harvesting Applications. Solar Energy Materials and Solar Cells, 145, 432-439. https://doi.org/10.1016/j.solmat.2015.11.014

[21] Bett, A., Eisenlohr, J., Höhn, O., Bläsi, B., Benick, J., Repo, P., Savin, H., Goldschmidt, J. and Hermle, M. (2014) Front Side Antireflection Concepts for Silicon Solar Cells with Diffractive Rear Side Structures. 2014 29th European Photovoltaic Solar Energy Conference and Exhibition, Proceedings of the International Conference, Amsterdam, the Netherlands, 22-26 September 2014, 987-991.

[22] Kang, G., Yoo, J., Ahn, J. and Kim, K. (2015) Transparent Dielectric Nanostructures for Efficient Light Management in Optoelectronic Applications. Nanotoday, 10, 22-47. https://doi.org/10.1016/j.nantod.2015.01.008

[23] Kim, J.J., Lee, Y., Kim, H.G., Choi, K.J., Kweon, H.S., Park, S. and Jeong, K.H. (2012) Biologically Inspired LED Lens from Cuticular Nanostructures of Firefly Lantern. Proceedings of the National Academy of Sciences of the United States of America, 109, 18674-18678. https://doi.org/10.1073/pnas.1213331109

[24] Schulze, M., Damm, M., Helgert, M., Kley, E.B., Nolte, S. and Tünnermann, A. (2012) Durability of Stochastic Antireflective Structures-Analyses on Damage Thresholds and Adsorbate Elimination. Optics Express, 20, 18348-18355. https://doi.org/10.1364/OE.20.018348

[25] Hobbs, D.S. and MacLeod, B.D. (2005) High Laser Damage Threshold Surface Relief Micro-Structures for Antireflection Applications. Proceedings of $2007 \mathrm{La}$ ser-Induced Damage in Optical Materials, 6720, 67200L. https://doi.org/10.1117/12.754223

[26] Weng, B., Qiu, J., Yuan, Z., Larson, P.R., Strout, G.W. and Shi, Z. (2014) Responsivity Enhancement of Midinfrared PbSe Detectors Using $\mathrm{CaF}_{2}$ Nano-Structured Antireflective Coatings. Applied Physics Letters, 104, Article ID: 021109. https://doi.org/10.1063/1.4861186

[27] Zhou, L., Dong, X., Zhou, Y., Su, W., Chen, X., Zhu, Y. and Shen, S. (2015) Multiscale Micro-Nano Nested Structures: Engineered Surface Morphology for Efficient Light Escaping in Organic Light-Emitting Diodes. ACS Applied Materials \& Interfaces, 7, 26989-26998. https://doi.org/10.1021/acsami.5b08575

[28] Du, Y., Liu, S., He, H., Jin, Y., Kong, F. and Guan, H. (2012) Laser-Induced Damage Properties of Antireflective Porous Glasses. Optics Communications, 285, 5512-5518. https://doi.org/10.1016/j.optcom.2012.07.120

[29] Thomas, I.M. (1986) High Laser Damage Threshold Porous Silica Antireflective 
Coating. Applied Optics, 25, 1481-1483. https://doi.org/10.1364/AO.25.001481

[30] Hobbs, D.S., MacLeod, B.D. and Sabatino, E. (2012) Continued Advancement of Laser Damage Resistant Optically Functional Microstructures. Proceedings of 2012 Laser-Induced Damage in Optical Materials, 8530, 853000.

https://doi.org/10.1117/12.976909

[31] Hobbs, D.S. and MacLeod, B.D. (2007) Design, Fabrication, and Measured Performance of Anti-Reflecting Surface Textures in Infrared Transmitting Materials. Proceedings of 2005 Laser-Induced Damage in Optical Materials, 6720, 67200L. https://doi.org/10.1117/12.604532

[32] Tulli, D., Hart, S.D., Mazumder, P., Carrilero, A., Tian, L., Koch, K.W., Yongsunthon, R., Piech, G.A. and Pruneri, V. (2014) Monolithically Integrated Micro- and Nanostructured Glass Surface with Antiglare, Antireflection, and Superhydrophobic Properties. ACS Applied Materials \& Interfaces, 6, 11198-11203. https://doi.org/10.1021/am5013062

[33] Greene, J.M. (1992) Locating Three-Dimensional Roots by a Bisection Method. Journal of Computational Physics, 98, 194-198. https://doi.org/10.1016/0021-9991(92)90137-N 